\title{
A 3-year disease free survival in a patient with metastatic pancreatic adenocarcinoma following folfirinox chemotherapy: A case report.
}

\author{
Ahmed Ayad", Daniela Urueta, Wajahat Dawood, Neelam Asghar, Julio Peguero \\ Research Department, Oncology Consultants, P.A. Houston, Texas, United States
}

\begin{abstract}
Metastatic pancreatic adenocarcinoma confers a poor prognosis. Even with recent treatments, complete response of the disease is still very rare. In this case report, we present the case of a 67-yearold male with metastatic pancreatic adenocarcinoma who achieved a complete response lasting for more than three years after receiving induction chemotherapy with FOLFIRINOX for 16 months followed by same regimen maintenance for one year. Therefore, this evidence-based approach is recommended for highly selected patients with sustained responses to chemotherapy.
\end{abstract}

Keywords: Pancreatic adenocarcinoma, Chemotherapy, FOLFIRINOX, Gemcitabine, Survival.

Accepted on 11 June 2018

\section{Introduction}

Pancreatic cancer is the fourth common cause of cancer deaths in the United States with a mortality rate of 10.9 per 100,000 population during the period of 2011-2015 [1]. According to the American Cancer Society, It is estimated that 55,440 persons will be diagnosed with pancreatic cancer and 44,330 will die from it in 2018 [1]. This high mortality rate is, in part, because the majority of patients are first diagnosed at an advanced stage of the disease, making curative approach difficult to obtain [2]. Without treatment, the median survival rate is less than 6 months in metastatic settings, and 10 months in locally advanced settings [2]. The most common type of pancreatic cancer is adenocarcinoma (85\%), which arises from the ductal epithelium [1]. Gemcitabine has been the reference regimen for advanced pancreatic cancer until a study by Conroy et al. favored FOLFIRINOX (a combination of 5fluorouracil, oxaliplatin, irinotecan and leucovorin) with a median overall survival of 11.1 versus 6.8 months [3].

\section{Case Presentation}

A 67-year old African American male patient with a cigarette smoking history of 25 pack-year and occasional alcohol consumption, was seen in August 2013, with a 4-month history of mild to moderate, constant, dull epigastric pain, not radiated elsewhere, and aggravated by eating. The patient also experienced one episode of self-limited hematuria, for that he underwent cystoscopy with no abnormal findings. Patient reported fatigue, decreased appetite, weight loss of 40 pounds over the course of one year, and denied jaundice, nausea or vomiting.

An MRI of the abdomen was obtained and revealed multiple hepatic lesions. A PET CT scan showed FDG avid mass in the head of the pancreas and innumerable metastatic FDG lesions in the liver. A liver biopsy with ultrasound guidance done on August $23^{\text {rd }}, 2013$ confirmed the diagnosis of poorly differentiated metastatic adenocarcinoma of the pancreas. Next
Generation Sequencing of the tumor tissue showed a genomic alteration of the CDK 4 gene.

Further testing revealed markedly elevated levels of tumor markers consisting of CA 19-9 exceeding $1103 \mathrm{U} / \mathrm{mL}$ (normal range: 0-35 U/mL), CEA 64.8 (normal range: 0.0-4.7 U/mL) and normal liver functions.

After discussing treatment options with the patient, he started the first cycle of the FOLFIRINOX chemotherapy regimen on September $16^{\text {th }}, 2013$. Patient received a total of 35 cycles till mid-February 2015 with a 2 -week interval in between. Tumor response was evaluated every 8 weeks using PET CT of the abdomen and pelvis, or CT scans of the chest, abdomen and pelvis with and without contrast to assess lesion measurements as per RECIST 1.1 guidelines and tumor markers (Figure 1).

A PET CT scan performed on February $16^{\text {th }}, 2015$ showed no increased FDG activity suspicious of active neoplasia. A CT scan of the abdomen and pelvis on August 17 $17^{\text {th }}, 2015$ showed stable chronic changes with complete response of pancreatic and hepatic lesions. Tumor markers showed dramatic decrease to normal levels; with CA $19-9$ of $26 \mathrm{U} / \mathrm{mL}$ and CEA of 2.2 $\mathrm{U} / \mathrm{mL}$.

The patient continued on maintenance therapy with the same chemotherapy regimen and same tumor assessment schedule for one year (Figure 2).

With a strong evidence of biomarkers and radiological complete response, patient received the last cycle of chemotherapy and discontinued the FOLFIRINOX on April, $25^{\text {th }}$ 2016. After completing FOLFIRINOX, patient continues to follow up at clinic every three months with no clinical or radiological signs of recurrence.

Patient experienced mild hypercalcemia since his presentation until now, and transient elevation of alkaline phosphatase that resolved after the discontinuation of chemotherapy. A nuclear scan of the parathyroid gland was done on December $17^{\text {th }}$, 
2017 and showed no definite scintigraphic evidence of a parathyroid adenoma.

Most notable toxicities during the period of active treatment were grade 1 to grade 3 neutropenia managed with Pegfilgrastim, grade 2 neuropathy, and grade 1 alopecia; all could be chemotherapy-induced toxicities, mainly the Oxaliplatin. These toxicities have resolved gradually after the discontinuation of chemotherapy.

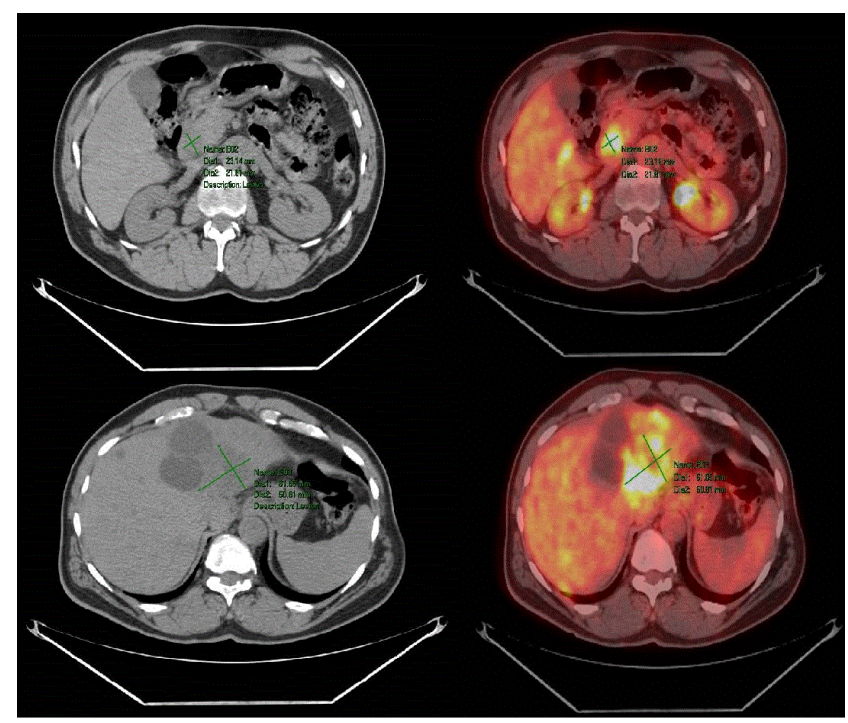

PET/CT scan September 2013

Figure 1. Primary lesion (head of pancreas) and metastatic lesion (left liver lobe).

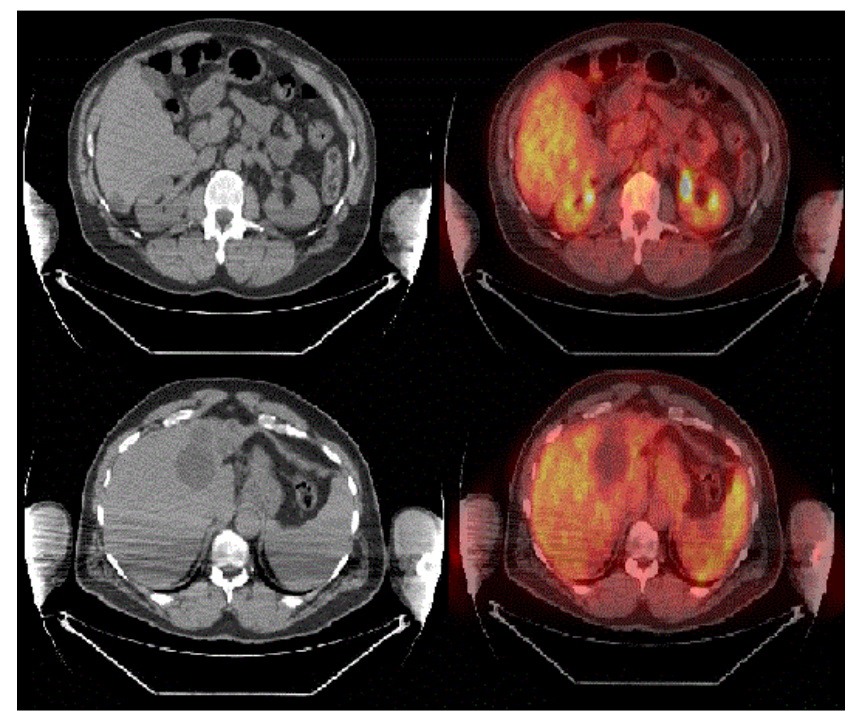

PET/CT scan February 2015

Figure 2. Complete response of all lesions.

\section{Discussion}

For over a decade, Gemcitabine has been the recommended palliative treatment for metastatic pancreatic cancer with an average response rate of $9 \%$ and low survival benefits [4]. In 2011, Conroy et al. [3] compared FOLFIRINOX to Gemcitabine in the palliative treatment of metastatic Pancreatic cancer. Results showed a significant increase in the median overall survival of 11.1 months for FOLFIRINOX compared to 6.8 months for Gemcitabine. In addition, progression-free survival for FOLFIRINOX was higher compared to Gemcitabine (6.4 versus 3.3 months). Lastly, in terms of tumor response rate, FOLFIRINOX showed a higher rate of $31.6 \%$ compared to $9.4 \%$ for Gemcitabine [3].

Regarding the safety profile, Conroy et al. [3] concluded that FOLFIRINOX is less favorable than Gemcitabine. Relatively higher rates of grades 3 and 4 neutropenia, febrile neutropenia, thrombocytopenia, and grade 2 alopecia were observed with FOLFIRINOX compared to Gemcitabine. However, most of the grades 3-4 complications related to FOLFIRINOX were resolved by dose reduction [3].

In a retrospective cohort of 50 patients with locally advanced or metastatic pancreatic cancer treated with FOLFIRINOX, $40 \%$ of patients had grades 3 and 4 neutropenia, and $8 \%$ developed neutropenic fever. In addition, patients had grades 3 and 4 toxicities of thrombocytopenia, diarrhea, fatigue, nausea, and vomiting [4]. In contrast, our patient had relatively lower toxicities of grade 1 to grade 3 neutropenia that was successfully managed with Pegfilgrastim, grade 2 neuropathy, and grade 1 alopecia.

Complete response is a rare outcome in patients with metastatic pancreatic cancer. Out of 171 patients receiving FOLFIRINOX, only one patient $(0.6 \%)$ had a complete response [3]. In our patient, a complete response was achieved after 35 cycles of FOLFIRINOX over 16 months.

Similar to Conroy et al. [3] study, our report supports the use of FOFIRINIX as the first-line regimen for metastatic pancreatic cancer in patients younger than 65 years with a good performance status (ECOG 0 or 1 ) and normal bilirubin levels.

\section{Conclusion}

To our knowledge, we present the first case of a patient with metastatic non-resectable adenocarcinoma of the exocrine pancreas to achieve a complete response and continues to be on a 3-year disease free survival after monotherapy with FOLFIRINOX.

\section{Acknowledgement}

We wish to thank Julio Peguero MD, Randall Stenoien MD, Luis T. Campos MD for providing editorial support.

\section{Conflict of Interest Disclosure}

Dr. Peguero serves in the speaker bureau for Foundation Medicine, Bristol Myers Squibb, and Bayer. He directs phase 1 and 2 clinical trials, funded by various pharmaceutical companies, within the Oncology Consultants Research Department. None of the remaining authors have any conflict of interest.

\section{References}

1. Society AC. Cancer facts \& figures 2018. Atlanta: American Cancer Society; 2018. 
Citation: Ayad A, Urueta D, Dawood W, et al.. A 3-year disease free survival in a patient with metastatic pancreatic adenocarcinoma following folfirinox chemotherapy: A case report. J Med Oncl Ther 2018;3(1):18-20.

2. Deeb A, Haque SU, Olowokure O. Pulmonary metastases in pancreatic cancer, is there a survival influence? J Gastrointest Oncol. 2015;6:48-51.

3. Conroy $\mathrm{T}$, Desseigne F, Ychou M, et al. FOLFIRINOX versus gemcitabine for metastatic pancreatic cancer. $\mathrm{N}$ Engl J Med. 2011;364:1817-25.

4. Rombouts S, Mungroop T, Heilmann $\mathrm{M}$, et al. FOLFIRINOX in locally advanced and metastatic pancreatic cancer: A single centre cohort study. J Cancer. 2016;7:1861

\section{${ }^{*}$ Correspondence to:}

Ahmed Ayad

Research Department, Oncology Consultants

P.A. Houston, Texas

United States

E-mail: aayad@oncologyconsultants.com 